\title{
Overlapping Generations Models and Graded Age-Set Societies
}

\author{
by \\ MERWAN H. ENGINEER AND LINDA WELLING *
}

From Africa through North America to Asia, anthropologists have found societies with formalized age-group systems. In these societies, social and economic relations between individuals are regulated by well-established rules governing transitions through the lifecycle. In this paper we detail the relationship between the classic formulations in STEWART's (1977) Fundamentals of Age-Group Systems and the overlapping generations (OLG) model. We establish that OLG models can accurately represent a large subset of actual age-group societies called graded age-set societies. Thus, an OLG model bears a close resemblance to reality. (JEL: J10, P00, Z10)

Keywords: Age Sets, Graded Age-Group Systems, Lifecycle, Overlapping Generations Model.

\footnotetext{
*We thank Eric Roth for introducing us to Stewart's work and helping us understand age-group societies, and two anonymous referees for their further refinements. We are grateful to Francisco Gonzales, Ming Kang, and seminar participants at the Canadian Economics Association Meetings 2000, Society for Economic Dynamics Meetings 2000, the University of Guelph and the University of Victoria for their suggestions. We thank Marie-Karin Godbout and Ryan George for their very able and enthusiastic research assistance. Finally, we thank the Social Science and Humanities Research Council of Canada for financial assistance. We are responsible for any remaining errors.
} 


\section{Introduction}

The overlapping generations (OLG) model is arguably the paradigm general equilibrium model in economics. It captures the lifecycles of individuals and market incompleteness, essential features of real economies. In a typical OLG model, an individual's life span is divided into two, or possibly three, stages. Each stage differs from the other(s) by the economic choices open to the individual in that stage: savings/consumption; work/leisure. An economic model consisting of citizens in different stages, simultaneously consuming and producing, generates a dynamic path for the economy as a whole.

Economists view OLG models as stylized models with each period of life describing a general "stage of life". Typically, the periods are of the same discrete length and agents within a "generation" are synchronized in their transitions through various stages of life. In this literature "generation" refers to an age cohort; kinship relations between these "generations" are often ignored because the focus is on the economy as a whole. ${ }^{1}$ When kinship patterns are examined, they are modeled in a highly stylized way so as to retain the age cohort structure: all members of a "generation" are in the same age-cohort. This homogeneity is imposed to make the model analytically tractable.

Though the model has provided numerous theoretical insights, it is difficult to apply directly because in most modern economies members of age cohorts are not synchronized in their transitions through various stages of life. Most applied researchers have avoided the daunting task of tracking the lifecycles of heterogeneous age cohorts and have relied on simpler models. ${ }^{2}$ Unable to point to any actual societies that match the stylized model, economists tend to view and teach the OLG model as a useful fiction.

This paper establishes that the stylized OLG model captures key features of actual societies as described by anthropologists. These societies are found around the world and belong to what ethnographers call age-group societies. Such societies have been found in Africa, North America, South America, Central Asia, Taiwan, New Guinea, Switzerland and Albania. Africa has the greatest number and variety of well-documented systems [RITTER [1980], STEWART [1977]]. In these societies, social relations between individuals are governed by rules that dictate and constrain behaviour within and between members of age groups as they pass in sequence through the various steps in the social hierarchy. SPENCER [1997] describes the overarching premise of such societies as the respect for age. Other fundamental premises are honor, associated with the integrity of kinship, and purity, associated with status and caste.

In these real age-group societies, individuals (often only males) are collected into groups by age-cohort. New groups are initiated at regular intervals and members of any group are of roughly similar ages. Allocations at any point in time are determined by the

\footnotetext{
1 AZARIADIS [1993, xii], the leading OLG graduate text, states: "Overlapping generations models... are made up of unending sequences of heterogeneous finitely-lived households with weak or non-existent dynamic links". Thus, explicit father-son linkages are ignored.

${ }^{2}$ Notable exceptions are GOKHALE ET AL [2001], and the references on generational accounting therein. This literature began with AUERBACH AND KOTILIKOFF [1987] who simulate a 58generation OLG model of the United States economy. In their model, a period corresponds to one year, and agents live for 58 periods so that 58 "generations" are alive at any time.
} 
age-cohort's position in the age-grade system. An individual's position in the age-grade structure governs work assignment, marriage possibilities, parental relationships and property inheritance. If females are not initiated into an age group, their positions are determined by their affiliation with males: in many tribes, a girl is assigned to her father's age group before marriage and switches to her husband's upon marriage. Compared to modern market societies, prices play a smaller role in allocating people and resources.

The definitive analytical work in this area is Fundamentals of Age-Group Systems by STEWART [1977]. Of this work SPENCER [1997, 94] says: "This provides a pioneering and unsurpassed attempt to place a world-wide range of these systems within a logical framework, devising a method of analysis and identifying the implications of the rules that characterize them." Stewart's book details rules on the age-group model and the age-grade system as well as on societies incorporating both of these rules called the graded age-group system. Age groups divide the principal actors of a society into cohorts of roughly similar age - like the generations of an OLG model - whereas age grades detail the stages of the lifecycle. In a society with a graded age-group system, individuals are grouped into cohorts of approximately the same age and a cohort proceeds through the grades in sequence. ${ }^{3}$

Stewart develops an empirically relevant prototype model that we term a standard graded age-set system. He describes other age-group models as variants of this system. As economists we were struck by the similarities between Stewart's systems and the OLG model. Our investigations revealed exact correspondences between the models.

In this paper we provide four propositions which detail the relationship between the OLG model and the classifications in Stewart. We define a standard OLG system and show that this system is also an age-set model (see Table 1), an age-grade system (see Table 2), and a graded age-set system. We further show that the standard graded age-set system is equivalent to the standard OLG system with an explicit participation rule. Thus, the well-known properties of the OLG model can be applied to modeling actual societies.

Stewart's work points the way to using the OLG model as a serious demographic framework. His work provides "recruitment" rules for collecting individuals of disparate ages into generations. He also creates a taxonomy of social systems based on deviations from the prototype. Our work provides a link with Stewart's and generates additional insights and questions about the workings of graded age-group societies.

The paper proceeds first to define the standard OLG system. Section 3 presents Stewart's age-set model and age grade system. These systems are compared to the OLG system in Section 4. Sections 5 and 6 extend the comparison to variants of the graded age-set system. Section 7 concludes.

\footnotetext{
3 The different dimensions are neatly captured in discussions of inequality in these societies. BERNARDI $[1985,30]$ argues that while at any point in time an age-grade society can be seen as a gerontocracy, from a dynamic perspective the orderly progression of age groups through the grades can be seen to guarantee full equality to all members of the system.
} 


\section{Overlapping Generations Models}

The overlapping generations (OLG) model originated with ALLAIS [1947] and SAMUELSON [1958]. ${ }^{4}$ Distinct from the Arrow-Debreu model, not all agents can interact with each other - agents are "born" and "die" and therefore have temporal existence. Thus, markets are incomplete. For this reason, HAHN AND SOLOw [1995] prefer the OLG model to the Arrow-Debreu model in macroeconomics, and the former can arguably be thought of as the paradigm general equilibrium model in economics.

AUERBACH AND KOTLIKOFF's [1998, 33] undergraduate text description of the OLG model is typical:

Inhabitants of this country live for just two time periods. In their first period, they are young and go to work. In their second, they are old and retired. At the end of their second period, they pass away. All individuals born at the same time are identical.

Although each generation dies after two periods, the economy is ongoing. At the beginning of each period, a new generation is born, and the previous young generation passes from youth into old age. Hence, in each period, there is always a set of young people and a set of old people.

A period of time in our model refers, in real time, to roughly 30 years of adulthood. You should think of each generation's "youth" as corresponding to the 30 years between ages 20 and 50 and its old age as corresponding to the 30 years between ages 50 and 80 .

The quotation depicts a stylized society where a new generation of identical economic agents is born at the beginning of each period; each generation lives two periods and dies at the end of the second period of life. ${ }^{5}$ Agents are born into this model universe as young adults like Athena sprung full-grown from the mind of Zeus. The framework is motivated, but not modeled, as representing a situation where individuals enter the economy at chronological age 20 and live two thirty-year periods before dying.

After explaining the demography, Auerbach and Kotlikoff describe utility, production and markets. Figure 1 provides a time schematic of this economy. The beauty of the OLG model is that it not only details each agent's lifecycle (explained in Figure 1) but also specifies the interactions between agents at different points in their lifecycle. For example, in period 2, generation 1 is old and sells (dissaves) all its capital stock to buy consumption goods. In the same period, generation 2 agents are young and buy the capital stock from their elders to save for their old age (in period 3).

\footnotetext{
${ }^{4}$ AZARIADIS [1993], BROCK [1990] and GEANAKOPOLOS [1987] survey theoretical applications of the model. The model has been expanded into areas of interest to the other social sciences, such as gift-giving/inheritance (BERNHEIM, SHLEIFER AND SUMMERS [1985], KARATZAS, SHUBIK AND SUDDERTH [2002]), marriage and divorce (GREENWOOD, GUNER AND KNOWLES [2003]), and human capital acquisition and childcare (KENNEDY AND WELLING [1997]).

${ }^{5}$ This demography suffices to capture incomplete markets in OLG models. Economists mostly use the OLG model to explore the implications of incomplete markets; see BULLARD [1992].
} 
(Figure 1 here)

This structure is the simplest possible characterization. In this prototype model all agents are purely selfish and all transactions take the form of trades rather than gifts. The old sell all their capital and bequeath none to the next generation because this is optimal behavior for purely selfish agents. Agents are "born" into the economy in every period but there are no explicit kinship links. The simple model can be expanded in a number of ways. Models have been developed with $N$-period lived agents, where $N$ can be uncertain and/or infinite (see GEANAKOPOLOS [1987]).

The age structure is the distinguishing feature of OLG models. In almost all OLG models, a stylized demography provides the structure for the time periods. The usual convention is to index agents by the time period in which they first appear in the economy, or "are born". Thus, "generation $j$ " agents are born at the beginning of period $j$. In subsequent periods, this group of agents is identified as generation $j$ so long as they live; once they die, the generation disappears from the model. As agents live $N>1$ periods, living generations overlap at any point in time. ${ }^{6}$

A period index and an age index are used to track generations. Let $A_{a t}^{j}$ denote the group of agents from generation $j$ who are age $a$ at the beginning of period $t$; the index $a=t-j+1$ gives the age of the agents in terms of the number of periods they have been in the model with $1 \leq a \leq N$. Hence, those born in period $t$ are age 1 . Those who die at the end of period $t$ are age $N$ : they were born $N-1$ periods prior to the period $t$ generation and are from generation $j=t-(N-1)$. At the beginning of period $t$, the set of living agents is $A_{t}=\left\{A_{a t}^{t-a+1}\right\}_{a=1, \ldots, N}$.

The above features are common to most OLG models. As we could not find a general description in the literature, we formalize and integrate these features in the following definition.

Definition. The standard OLG system consists of the following six elements.

1. (Time) Time is partitioned into discrete periods of equal length. Periods are indexed by whole numbers.

2. (Agents and Generations) At the beginning of period $t$, a new group of agents, denoted by $A_{1 t}^{t}$, is born; the subscript $a=1$ indicates that this is the first period of the agents' lives and the superscript $j=t$ indicates that they belong to generation $t$. Thus, agents are assigned a generation number that directly corresponds with their birth period. The longest-lived member of generation $j$ lives $N>1$ periods and leaves the model at the end of period $j+N-1$ (in a perpetual economy). Population changes only by "birth" and "death". This is a "closed economy" where there is no migration.

\footnotetext{
${ }^{6}$ As explained in the introduction, economists use the term "generation" to refer to an age-cohort, and kinship links between agents of different generations are usually not explicit. The exception is the bequest literature that examines the transmission of wealth in a dynamic economy. This literature assumes that all children of a particular generation are in the same age cohort. Often only one child is considered; multiple children and intra-generational marriage are modeled by BERNHEIM AND BAGWELL [1988].

${ }^{7}$ OBSTFELD AND ROGOFF [1996] reference OLG models with two or more economies.
} 
3. (Endpoints) A perpetual economy has neither a beginning nor an end and the demographic structure is completely described by elements 1 and 2 above. However, if the economy has a beginning or end, or both, the demography must be specified at the endpoint(s). Consider an ongoing economy with a beginning period $\tau>-\infty$ but no end. Here, the usual specification is that the economy begins with a full cross-section of generations, $A_{\tau}=\left\{A_{a \tau}^{\tau-a+1}\right\}_{a=1, \ldots, N}$, representing the complete lifecycle; $\left\{A_{2 \tau}^{\tau-1}, A_{3 \tau}^{\tau-2}, \ldots, A_{N \tau}^{\tau-N+1}\right\}$ are often described as the "pre-existing agents". Such an ongoing economy is a perpetual economy from time $\tau$ onward. Finally, the economy can have a finite endpoint $T<\infty$. In this case there is no convention for specifying the demography after period $T$. Hereafter, we assume a perpetual economy unless otherwise noted.

4. (Period length) The discrete time intervals correspond to periods during which agents from at least one living generation face a substantive choice or life passage. Each period is short enough that all relevant choices or life passages are captured.

5. (Lifecycle stages) The lifecycle is a sequence of $C \geq 2$ distinct and totally-ordered stages denoted $\left\{\Sigma_{\sigma}\right\}_{\sigma=1, \ldots, C} .{ }^{8}$ Agents enter $\Sigma_{1}$ and progress through the lifecycle. Each lifecycle stage spans at least one whole period, so $C \leq N$. Stages $\Sigma_{1}, \ldots, \Sigma_{C-1}$ consist of whole periods or multiples thereof. The typical agent dies in the final stage so $\Sigma_{C}$ need not be of integer length. Stages are contiguous; if a stage consists of two or more periods, these periods are contiguous. In each period, the assignment of stages to agents is a weakly increasing function of each agent's age. Thus, an agent of age 1 is in $\Sigma_{1}$ and an agent of age $N$ is in $\Sigma_{C}$.

6. (Stationarity) In the standard OLG system, there is a unique mapping from agent age to lifecycle stage: $\Sigma(a):\{1, \ldots, N\} \rightarrow\left\{\Sigma_{1}, \ldots, \Sigma_{C}\right\} .^{9}$ This mapping is independent of the time period. Since a new generation is born in every period and every generation $j$ advances in age according to $a=t-j+1$ until $a=N$, there is always a one-to-one correspondence between age and the set of living generations; that is, the cross-section of generations by age is stationary. It then follows from the uniqueness of $\Sigma(a)$ that the cross-section of generations by stage is also stationary. (For an arbitrary $t$, the assignment of living generations $j=t-N+1, \ldots, t-a+1, \ldots, t$ to stages is constant: $\Sigma_{\mathrm{C}}=\Sigma(N), \ldots, \Sigma_{\sigma}=$ $\Sigma(a), \ldots, \Sigma_{1}=\Sigma(1)$ respectively.

Elements 1-3 are the bare bones description of the standard OLG system. Elements 4-6 describe how the system is used to address substantive economic issues. Element 4 simplifies modeling by keeping the number of periods to the minimum required to

\footnotetext{
${ }^{8}$ BLANCHARD's [1985] continuous time OLG model is a notable exception. All living agents have an equal probability of dying in the next interval of time. Agents are replaced (born into the economy) at the same rate as agents die. This OLG model does not capture standard features of the lifecycle where most agents can expect to live through established life passages.

${ }^{9}$ Since $C \leq N$, two or more ages may map into the same stage. Hence, the function $\Sigma(a)$ is not generally invertible (i.e. there is no unique mapping from stage to age).
} 
capture the relevant activities. Element 5 describes the existence of life stages for each agent. Economists do not use the term "stages". Instead, more descriptive terms like youth, student, working age, retirement etc. are typically used. Where such language is not used, stages are implicit. Element 6 describes the stationary lifecycle structure of the system. Stationarity enables us to model an essentially dynamic system in terms of a representative individual and representative time period.

The elements of the standard OLG system are found in most OLG models. A particular OLG model details all the characteristics of each agent at each age and stage through the specification of endowments, preferences, technology, information, and societal constraints. The solution for the model fully describes the interactions between and within the overlapping generations.

\section{Age Sets and Age Grades}

STEWART [1977] develops a formal analysis of age-group systems, drawing on ethnographic studies of societies across the world.

\subsection{The Age-Set Model}

The original definition of an age set comes from RADCLIFFE-BROWN [1929]: "(a) recognized and sometimes organized group consisting of persons... who are of the same age... Once a person enters a given age-set, whether at birth or by initiation, he remains a member of the same set for the remainder of his life." STEWART [1977,2] says a society "has an age-set system when it has a number of age sets with no members in common and distinct mean ages." The age difference between the youngest and oldest members of any single group is inversely related to the frequency of initiation.

Stewart defines an age-set model in terms of eight formal characteristics. ${ }^{10}$ The characteristics reveal that age sets are much like generations in the OLG model: they are totally ordered, non-overlapping, groups of individuals of roughly the same age. Membership in an age set identifies an individual with his generational cohort.

\subsection{The Age-Grade System}

(Table 1 here)

In contrast to age sets, age grades identify individuals' social roles according to their stage in the lifecycle. For example, elders usually have very different rights and responsibilities from those available to warriors. Stewart identifies these collections of rights and responsibilities with rule-sets and provides the following list of constraints on the transition rules that govern the assignment of rule-sets to individuals or groups.

(Table 2 here)

${ }^{10}$ FONER AND KERTZER [1978] use different criteria to designate age-set societies and use a definition much like Stewart's definition of graded age-group systems. STEWART [1977] discusses earlier definitions. 
Stewart's formal rules capture the main features of age-set and age-grade societies in both a parsimonious and precise way. As with all generalizations, there is a trade-off between providing a simple representation and losing some details. An obvious example is the no-interval constraint, which assumes that the ceremonies of assignment and disassignment are simultaneous. In some societies, they may be concurrent; in others, there may be an interval between an age set's disassignment from one grade and its assignment to the next. The generalization is useful to the extent that nothing significant occurs in this interval.

Later, we link age sets with age grades and define a graded age-set system.

\section{Comparisons of Economic and Ethnographic Systems}

Clearly, there are similarities between Stewart's systems and OLG models. In particular, economists' generations resemble age sets and the common lifecycle stages resemble age grades. In this section, we formally show that Stewart's systems are general enough to include the "economy" constructed by the standard OLG system.

\subsection{Age Sets and the OLG System}

Proposition 1. The standard OLG system is an age-set model.

Proof. To prove this proposition, we show that the standard OLG system satisfies each of Stewart's eight characteristics in Table 1.

(1) In an OLG model, a group is a generation. A new generation $j$ is formed at the beginning of each new period $j$. Generation $j$ consists of all agents born into the economy in period $j$. As periods are discrete, there is a total ordering on these groups. (In an economy with an end period $\mathrm{T}<\infty$, the ordering is complete up to $\mathrm{T}$. In an economy with a beginning period $\tau>-\infty$ and no "pre-existing" agents, the ordering is complete from $\tau$ onward. With a full cross-section of pre-existing agents $A_{\tau}=\left\{A_{a \tau}^{\tau-a+1}\right\}_{a=1, \ldots, N}$, there is a total ordering on all groups.)

(2) Recruitment is sequential and hence non-overlapping. (It is the generations that overlap, not the recruitment into generations.)

(3) By construction, any OLG model has at least two generations/groups. (These overlap as the name implies).

(4) In the OLG system, group $j$ dissolves when the last member of generation $j$ dies. At least one member of each generation lives $N$ periods; hence, no generation disappears from the model before the previous generation.

(5) In the OLG system agents enter the economy at "birth", which occurs at the beginning of a period. Hence, both the minimum enrolment age and the basic enrolment age are 1 .

(6) Follows from the explanation to (1).

(7) (i)This is true in the standard OLG system because it is a closed economy and agents cannot leave the economy other than by dying. (ii) This follows from (4): a group only dissolves when the last member of that group dies.

(8) Follows from the explanations to (4) and (7). 


\subsection{Age Grades and the OLG System}

Proposition 2. The standard OLG system is an age-grade system.

Proof. The standard OLG system is an age-grade system where $C=n$ and $\Sigma_{1}$ corresponds to $G_{1}, \Sigma_{2}$ to $G_{2}, \ldots, \Sigma_{C}$ to $G_{n}$. This construction explicitly establishes (1)-(6) of Stewart's definition listed in Table 2.

Individuals move through the sequence of grades in one-to-one correspondence with the contiguous lifecycle stages.

\section{The OLG System as a Graded Age-Set System}

STEWART [1977, 129] writes: "When age-groups are a prominent feature of the social organization of a society, they almost always operate in conjunction with age-grades." 11

Definition. A graded age-group system is an age-group system combined with an agegrade system that satisfies the integration constraint.

Definition. (Integration constraint): "An age-grade system is integrated with an agegroup system if the transition rules of the age-grade system are such that it never happens that a member of group $S$ is assigned a higher grade than a member of a group $S+k, k \geq$ 1." (STEWART $[1977,135])$

This constraint requires that an individual never be assigned a higher age grade than someone who belongs to a senior age group. The notation $S+k$ refers to the $k$-th age group inaugurated before $S$, and $S-k$ is the $k$-th age group inaugurated after $S$. The minus sign indicates a junior age group and the plus sign a senior age group.

The following proposition establishes that the standard OLG system belongs to a subset of the graded age-group systems.

Definition. The graded age-set system is a graded age-group system that satisfies the ageset model.

Proposition 3. The standard OLG system is a graded age-set system.

Proof. The standard OLG system satisfies the integration constraint because lifecycle stages coincide with grades and all agents from a generation transit the lifecycle stages together. Thus, this proposition follows directly from Propositions 1 and 2.

\footnotetext{
${ }^{11}$ BERNARDI [1985, 3] concurs: "The formation of class and promotion in terms of grades are two aspects of the same phenomenon ... the recruitment of candidates to a class implies, in fact, their promotion to an initial age grade. Thus the relationship that is set in motion by recruitment and promotion not only brings about the structure of a special grouping; it also implies the chronological succession of classes in the grades ... ." STEWART [1977,142] states that the integration constraint is hardly ever violated. Violations occur in societies where age grades are independent of the age-set system, for example the Banapas Bedik and the Kikuyu.
} 


\section{The Graded Age-Set System as an OLG System}

We now seek conditions under which the standard OLG system can be used to describe a graded age-set society. This is a more ambitious task for two reasons. First, the graded age-group system can represent a bewildering variety of societies. Stewart simplifies the graded age-group system to an empirically important prototype and uses this as the reference for societies that vary from it. We refer to this prototype as a standard graded age-set system and use it as the basis for our analysis.

Second, the standard OLG system depicts a stylized model where "births" are perfectly synchronized across agents in each particular generation. To map to this model from a real society, we need criteria determining when real individuals living in chronological time exist as agents in the model. To apply the standard OLG system to a particular graded-age set society, we append to the former a "participation rule" that captures the enrolment and recruitment characteristics of that society. This expanded standard OLG system then captures the features of the graded age-set characterization of that society.

\subsection{Constraints and Level Rules for Simplifying Graded Age-Set Systems}

Graded age-set systems can display complicated patterns: more than one age set and/or grade sequence, sequences with time gaps, and individuals belonging to more than one group and/or sequence over time. ${ }^{12}$ STEWART [1977, 29] examines societies with these features, but notes that: "Very often there is only one sequence so that the system and sequence are identical." STEWART [1977, 121] notes that: “... sequence assignment rules are always, or almost always, such that an individual cannot join more than one sequence". His prototype is a system with a single sequence without gaps.

Based on empirical observation, Stewart introduces two additional constraints.

Definition. The group-unity constraint requires that all members of a group be at all times assigned the same grade.

Definition. The grade-filling constraint requires that each grade in the sequence be always occupied.

Though he provides examples of societies that violate these constraints, ${ }^{13}$ Stewart observes that violations are uncommon. He refers to these constraints together with the integration constraint as the three combination constraints. "[S]ystems that meet all three combination constraints are found around the world." (STEWART [1977, 143])

\footnotetext{
${ }^{12}$ BERNARDI [1985, 132-142] discusses six societies where the sexes belong to different groups and, thus, different sequences. In three, the female groups are assimilated into the men's so there is effectively only one sequence. The definition allows membership in more than one sequence and group but only one group in each sequence; i.e., a society may have sequences that have time gaps and do not overlap, and individuals may alternate between groups in these sequences.

${ }_{13}$ Both the Masai and the Borana Galla violate the group-unity constraint. The grade-filling constraint can be violated in societies with complex level rules (see next footnote).
} 
By the group-unity constraint, all members of a single age set are assigned the same grade. Observe that more than one age set can simultaneously inhabit an age grade. If so, the age sets are assigned the grade at different times with the earlier entrants disassigned first. Together with the integration constraint, this ensures that only adjacent age sets inhabit the same grade, and junior age sets are never assigned higher grades.

The grade filling constraint requires that each of grades $G_{1} \ldots, G_{i}, \ldots, G_{n}$ be occupied by at least one age set at all times. Thus the grade structure is stationary over time: for any two points in time, those members of the society in the age-grade system can be partitioned into the same grade structure.

Though the grade structure is stationary, the combination constraints are not sufficient to ensure that the graded age-set structure is stationary over time. For example, a grade may be occupied by one group now and two groups in the future.

To develop a more specific transition rule, Stewart introduces level numbers. When newly enrolled, an age set is assigned level number 1. This age set is assigned level number 2 when the next age set is enrolled, and so on. Thus, the level number for an age set tracks the number of enrolments since the age set was inaugurated. In graded age-set societies, the enrolment into an age set usually marks an important life passage and hence coincides with a new behavior rule set: (STEWART $[1977,145]$ ) “...a single ceremony or set of simultaneous ceremonies marks both the inauguration of a new group and any shifts in grade that this necessitates. This is indeed what happens in the great majority of systems with level rules." Level rules map from level numbers to age grades.

Definition. "A level rule is a transition rule that assigns a grade to an age group or disassigns one from it according to the level number of that age group. A level rule that assigns a grade is necessarily accompanied by one that disassigns the preceding grade (except in the case of the entry rule); and a level rule that disassigns a grade is necessarily accompanied by one that assigns the next grade (except in the case of the exit rule)." (STEWART [1977, 136])

Definition. "If an age-grade system has only one sequence of level rules, then that system uses simple level rules.” (STEWART [1977, 137])

With simple level rules, the mapping from level numbers to grades is unique but not necessarily complete. STEWART [1977, 135] states: “...simple level rules are overwhelmingly the most common type of transition rule. All the Taiwanese systems for which I have information, and indeed almost all the non-African systems that I know of, operate solely with simple level rules (except that some have exit rules that are not level rules, e.g., where the last grade is disassigned only at death)." 14

Stewart presents the Changki Ao Nagas society as an example of his prototype society. This society can be described by simple level rules where the level number $x$ assigns each age set the grade $x$, for $x=1, \ldots, 9$. Level numbers beyond 9 assign grade 9 . Figure 2 describes this assignment. The Changki inaugurate a new age set every 3 years.

\footnotetext{
${ }^{14}$ With complex level rules, different age sets will be assigned different grades for the same level number. This necessarily results in different numbers of age sets occupying grades at different points in time. Hence, the graded age-set structure is not stationary. STEWART [1977, 136] observes that complex level rules describe some societies in West Africa but nowhere else.
} 
The minimum and basic enrolment age is 12 and the nine grades are indicated by the Roman numerals in the boxes. Consider the situation when age set $S-11$ is enrolled. A cross-section of the society by grade is given by the bottom row. Age set $S-11$ is assigned level number 1 and grade I, age set $S-10$ is assigned level number 2 and grade II, and so on, until level number 9 is reached; thereafter age sets stay at grade IX.

\section{(Figure 2 here)}

As is evident from the figure, if all the transitions are governed by simple level rules, the combination constraints are satisfied and the number of age sets assigned to a given grade is constant over time. The only exception is the last grade, $G_{n}$, where the number of sets may vary when disassignment is by death or infirmity. Except for the last grade, the sequence of transitions through the grade structure is stationary in model time. When the inaugurations occur at constant intervals, the transition sequence is also stationary in real time.

\subsection{The Enrolment Characteristic and Recruitment Rules in Real Time}

Stewart describes "recruitment rules" consistent with the enrolment characteristic (rule 5 of the age-set model) for identifying how individuals of disparate ages are collected into age sets in real time. The recruitment rules are described using the timeline in Figure 3. This diagram identifies time intervals relevant to the inauguration of age set $S$ at date $d_{S} .{ }^{15}$ Age set $S$ cannot recruit members prior to this inauguration date. The length of the inauguration interval for age set $S, v_{S}$, is the length of the interval between the inauguration of $S$ and that of the next junior age set, $S-1$.

The length of the recruitment period for age set $S, r_{S}$, is the time interval during which members join this set. Though the enrolment ceremony may take a day or more, particular individuals are presented as being enrolled at a point in time in the recruitment period. Recruitment may be at a single ceremony at $d_{S}$ or continue through part of or the entire inauguration interval.

(Figure 3 here)

The length of the cessation interval for set $S, c_{S}$, is the time interval between the end of the recruitment period for the previous age set, $S+1$, and the end of the recruitment period for age set $S$. This interval is used to identify the individuals who are recruited into age set $S$. The enrolment characteristic requires that all individuals who reach the minimum enrolment age during $c_{S}$ join age set $S$ as soon as possible during its recruitment period. Thus, the maximum age difference between members of an age set is no greater than the length of $c_{S}$ and the bulk of enrolments take place in the first half of the inauguration interval. The shorter is $r_{S}$, the closer to $d_{S}$ are the bulk of enrolments.

15 STEWART [1977,113] says: "In fact, in all age-groups systems that I know of the rules governing the enrolment of an individual in a group are the precisely the same for all groups." Thus, this schematic is generally applicable. 
STEWART $[1977,38]$ notes that it is not uncommon for all members to be enrolled during a single ceremony at the beginning of the inauguration interval.

The following assumption describes a common recruitment rule.

Assumption: All inauguration intervals are of the same duration, and all enrolments occur at the beginning of inauguration intervals.

This recruitment rule implies that the maximum possible age difference within each age set is the length of the inauguration interval and age sets are formed by enrolling all members simultaneously at regular, discrete points in time. The assumption requires that inauguration dates be at regular intervals. STEWART [1977, 206-207] states that this is almost always the case in systems that determine inauguration dates by rules. In other "informal" inauguration systems, the evidence is that, in many cases, those who operate the system have a definite interval in mind. ${ }^{16}$ We use the above assumption in the next section and relax it in section 6.4.

\subsection{The Standard Graded Age-Set System and the Standard OLG System with the Participation Rule}

Definition. A standard graded age-set system is a graded age-set system that uses simple level rules to assign all grades at the beginning of inauguration intervals. Inauguration intervals are of constant duration and all recruitment takes place at the beginning of inauguration intervals.

The Changki Ao Nagas society (see Figure 2) is an example of a standard graded age-set system. Inaugurations take place every third autumn; all males between ages 12 and 14 are enrolled in their age set at the inauguration date. (MiLls [1926, 177-181])

The standard graded age-set system has the following essential features:

(i) There is a fixed (minimum and basic) enrolment age.

(ii) All enrolments take place at the beginning of inauguration intervals.

(iii) All inauguration intervals are of the same duration.

(iv) When an individual is enrolled into an age set he enters the first grade.

(v) Once in the grade system, an individual can be disassigned one grade and assigned the next grade only at the beginning of a new inauguration interval.

(vi) At any two dates, any two individuals who have passed the same time since their respective inaugurations are assigned the same grade.

Feature (iv) follows Stewart's description and provides a convenient reference interval with which to track groups transiting the grades. It also accords well with observation: enrolment ages are typically low. Often "real" grades start with inauguration around the age of puberty but putative grades like childhood are defined relative to it.

${ }^{16}$ FONER AND KERTZER [1978,1087-1090] point out that when transitions to different grades bring about different rights and powers, conflicts over the timing of transitions arise. These conflicts may be resolved within weeks, in which case we may view the inauguration interval as being effectively constant over time; when the resolution takes a much longer period of time, this assumption becomes less tenable. 
Features (i)-(v) impose the restriction that $G_{1}, G_{2}, \ldots, G_{n}$ are assigned to age sets at the beginning of regular inauguration intervals. A grade can be of any duration that is a multiple of the inauguration interval. However, the last grade $G_{n}$ need not be of integer length. Grades such as elderhood may encompass several inauguration intervals and may be open ended.

Whereas features (i)-(v) require individuals to transit grades at regular intervals, feature (vi) requires individuals in age sets to transit grades in the same way over time. Together these features yield an age-set and grade structure that is stationary over time.

The elements of the standard OLG system correspond closely to the above features. Regular inauguration intervals correspond to periods in OLG models. Individuals enrolled at the beginning of inauguration intervals into age sets correspond to agents born at the beginning of periods into generations. Assignment (and disassignment) of grades at the beginning of inauguration intervals corresponds to progression of generations through the lifecycle stages. The stationary age-set and grade structure mirrors the stationary generation and stage structure in the standard OLG system.

The standard OLG system lacks an element corresponding to feature (i), which captures the enrolment characteristic and recruitment rule. To apply the standard OLG system to a particular graded age-set society, we need a participation criterion determining when real individuals living in chronological time exist as agents in the model. This participation rule determines the transition, or initiation, of individuals from the society at large to members of a generation in the standard OLG system.

To establish equivalence between the systems, the participation criterion must capture the enrolment and recruitment characteristics of that society. We therefore append to the standard OLG system a seventh element that matches feature (i).

7. (Participation Rule) An individual is born into the economy at the beginning of period $j$ if he/she has reached the chronological "participation age" after the beginning of the (previous) period $j-1$.

The participation age is determined by the society at large; all active agents in the OLG model are drawn from individuals who pre-exist in the modeled society. We now show that the standard OLG system expanded to include the participation rule completely captures the graded age-set system characterization of real societies.

Proposition 4. The standard OLG system with the participation rule is equivalent to the standard graded age-set system.

Proof. We first show that the standard graded age-set system satisfies the seven elements that define the standard (perpetual) OLG system with the participation rule.

(1) (Time) Inaugurations partition real time into discrete periods of equal duration representable by whole numbers.

(2) (Agents and Generations) Enrolment in age set $S$ at the beginning of interval $v_{S}$ corresponds to agents being born at the beginning of period $j$ into generation $j$ in the OLG 
system. More generally, individuals in age set $S$ alive in interval $v_{S-k}$ correspond to generation $j$ agents in period $t=j+k, A_{a t}^{j}$, where $a=k+1=t-j+1$.

(3) (Endpoints) This is a perpetual system so endpoints need not be specified.

(4) (Period Length) The constant inauguration interval determines period length. For the enrolled age set, this is a substantive life passage. By features (iv) and (v), grades do not change within the period so no substantive life choices are omitted by using this discrete time unit.

(5) (Lifecycle) In the standard age-grade system, each individual passes through a sequence of $n>1$ distinct and totally-ordered grades $G_{1}, G_{2}, \ldots, G_{n}$. This sequence corresponds to agents passing through $C=n$ totally-ordered lifecycle stages where $G_{1}$ corresponds to $\Sigma_{1}, G_{2}$ to $\Sigma_{2}, \ldots, G_{n}$ to $\Sigma_{C}$. Let $N$ be the total number of inauguration intervals or, equivalently, the number of whole periods required for an age set to complete the grade sequence. Then, by feature (v), each grade (like each stage) lasts at least one whole period so $C=n \leq N$. If a grade lasts two or more periods, the periods must be contiguous. Periods are contiguous by (1). By the sequential assignment and nointerval constraints (see Table 2), grades (like stages) are contiguous. The grade sequence usually covers all $N$ whole periods.

(6) (Stationarity) Features (iii) and (vi) imply there is a unique mapping from age set to age grade according to the number of periods since enrolment, independent of the enrolment period $j$. When grades correspond to stages as in (5), this mapping is the same as in the standard OLG system, implying that the system's lifecycle structure is stationary.

(7) (Participation Rule) Feature (i) establishes a fixed enrolment age that gives the participation age for the standard OLG system. By (i), (ii) and (iv), all potential members of age sets enter the first grade at the beginning of an inauguration interval if and only if they have become eligible for membership after the last recruitment interval. Since all members of an age set are initiated at together and are immediately assigned the first grade, this satisfies the participation rule.

Thus, the standard graded age-set system is a standard OLG system with the participation rule. It remains to show the converse. First note that the participation rule implies the enrolment characteristic when all enrolments are at the beginning of the period. Since agents are born only at the beginning of a period, recruitment is at the beginning of an inauguration interval. The chronological participation age is the basic and minimum enrolment age.

The converse now follows immediately as a corollary to Proposition 3, as the participation rule does not change the unique and stationary mapping from generations to stages. Thus, the standard OLG system with the participation rule is a standard graded age-set system. This establishes equivalence.

This proposition establishes that a prototype graded age-set system and a prototype OLG system with a participation rule are isomorphic. Since this prototype system describes actual age-group societies, OLG systems bear a close resemblance to reality.

\subsection{OLG Models and Graded Age-Group Societies}


OLG models can also be used to describe more general age-group societies. We demonstrate this by relaxing each feature of the standard graded age-set system in turn.

The basic enrolment age of feature (i) is often a proxy for maturity. Indeed, Stewart presents a revised enrolment characteristic in terms of "level of maturity". To capture this with an OLG system requires a simple modification of the participation rule: replace the participation age with the level of maturity.

Relaxing feature (ii) allows for extending the recruitment period to a part of or the entire inauguration interval as described in Section 6.2. Capturing this extension with an OLG system requires a corresponding participation rule that allows individuals to be born into the economy as soon as they reach the age of participation, which may occur after the beginning of a period. ${ }^{17}$ Elements 2 and 5 of the standard OLG system must be rephrased to allow agents to be born after the beginning of a period and still complete stage 1 with their generation.

Relaxing feature (iii) allows inauguration intervals to be of different durations. The standard graded age-set system without regular inauguration intervals is stationary in model time (except for the number of age sets in the last grade). It follows that there is an equivalent OLG system in model time where age sets correspond with generations and grades with stages. Level numbers or, equivalently, periods measure duration.

Relaxing feature (iv) allows us to capture societies where grades begin before inauguration. To do this we assign level numbers to intervals prior to inauguration. Suppose age set $S$ is assigned the first grade in the interval $v_{S+e}$, where $e$ is the number of intervals to enrolment for age set $S$. We can now track all individuals who will be or are already enrolled in age set $S$ by the level number sequence $-e+1,-e+2, \ldots 1, \ldots N-e$, where $-e+1$ is the number corresponding to the first grade and 1 is the number for the grade assigned at inauguration. The correspondence follows by aligning generation $j$ with the level number $-e+1$.

Feature (v) is violated when the no-interval constraint is not satisfied. This is of minimal consequence when the interval between ceremonies for disassignment from one grade and assignment to another grade is short and/or when no substantive decisions are made in the interval. When a substantive decision is involved, the modeling issue is whether the decision can reasonably be assigned to one of the adjacent grades. If it can, we can apply the OLG model as above.

More generally, feature (v) is violated in societies that use "time rules". ${ }^{18}$ Time rules specify transitions between grades at times other than inaugurations. When the duration between transitions is constant, the analysis can be modified to replace inauguration intervals with time rule intervals. Except for the inaugurations, the time rules then describe transitions that can be captured by an OLG model.

Feature (vi) is violated by complex level rules that permit the number of age sets occupying a grade to vary over time. Stewart mentions that most often complex level

\footnotetext{
${ }^{17}$ The general participation rule in real time is: An individual is born into the economy as soon as possible in the real time interval $\left[d_{j}, d_{j}+\Delta\right]$ once they reach the chronological participation age (in the interval $\left[d_{j-1}+\Delta, d_{j}+\Delta\right]$ ), where $d_{j}$ is the (calendar) date of generation $j$ 's creation and $\Delta \leq d_{j}$ $d_{j-1}$ is the interval during which birth into generation $j$ is permitted. Date $d_{j}$ corresponds to the inauguration date $d_{S}$ and $\Delta$ is the duration of the common recruitment period, $r_{S}=r$.

${ }^{18}$ A few societies use time rules (see STEWART [1977,142]). Time rules can violate the nointerval constraint, in which case the above discussion applies.
} 
rules consist of cyclical sequences of level rules. For example, amongst the Nawdeba of Togo there are five level rules that are repeated in a cycle (STEWART [1977, 139-40]). The cyclical feature allows this society to be tracked by an OLG model in a straightforward manner. The grade assignment displays stationary periodicity.

Some societies use simple level rules that form an incomplete mapping from level numbers to grades; this also violates feature (vi). Some grades may be assigned on the basis of criteria other than age and hence the grade assignment is nonstationary. The nonstationarity will be localized as long as simple level rules bound the grades that are assigned on the other basis. If the other basis can be modeled, we have a complete temporal description of the grade structure.

Other more pronounced departures from the standard graded age-set system may be amenable to description by OLG models. For example, ENGINEER, ROTH AND WELLING [2004] use an OLG model to examine the Rendille of northern Kenya. This society deviates from Stewart's rules in a several ways, ${ }^{19}$ so much so that he does not consider them an age-set society though others do (e.g. BEAMAN [1981], BERNARDI [1985], ROTH [1993]). Nevertheless, the regular features of the society permit the application of OLG models to Rendille demography.

\section{Conclusion}

This paper details the demographic correspondence between the structure of the OLG model and Stewart's age-set, age-grade, and graded age-set systems. In particular, all three of Stewart's systems encapsulate the model society described by the standard OLG system. Conversely, the standard OLG system with an appropriate participation rule exactly describes the demographic structure of a large and important subset of actual graded age-group societies. Moreover, the OLG model can be readily generalized to capture many nonstandard features of actual graded age-set societies.

This paper provides a bridge between economics and ethnography. The literature on age-group systems is of interest to economists not only because it documents actual societies that fit the stylized OLG model, but also because it provides an analytical guide to the vast variety of age-group systems. In particular, STEWART [1977] follows the analytic method of building an empirically important prototype model and using this as a reference point by describing other systems in terms of their deviations from this prototype. His work is a set-theoretic taxonomic guide to an important class of human societies. It also illustrates a rigorous categorization of overlapping groups that is instructive of how to incorporate complex demography into economic OLG models.

We hope this paper will also serve as an introduction to OLG models for anthropologists, demographers, ethnographers and sociologists. Establishing the demographic correspondence between models is a necessary first step to using OLG models for analyses of populations, production and distribution. As we have discussed, economists have developed and solved a variety of stylized OLG models that focus on these features. These models and methods should prove fruitful for application to a variety of age-group and other societies. We have started this work with Eric Roth, an

${ }^{19}$ The particular feature of the Rendille discussed by STEWART [1977, 108-111] is the institution of "climbing". Climbing violates the age-set rules on no-resigning and no-overlapping because those who climb are initiated with their age mates but are enrolled into a more senior age set. 
anthropologist. In ENGINEER, Roth AND WeLLING [2004], we use an OLG model to examine the Rendille society of northern Kenya. We study the model's demographic dynamics given initial conditions for population and fecundity and the age-group rules. The computational model captures magnitudes, tracks lineages, and permits the counterfactual analysis of the impact of different age-group rules. Developing these connections between economics and anthropology may also provide insight into using the OLG framework to examine age structures as described in the sociology literature (e.g., RILEY, FONER AND WARING [1988]).

In more general terms, age-group societies are fascinating in themselves. They provide a revealing glimpse of archetypical social relations and suggest many questions. Why do societies organize themselves along age group lines? Are age-group systems institutions for controlling the young or restricting population or accommodating the ecology? Do individuals in age groups form natural coalitions? What types of age groups and institutional structures are stable? ${ }^{20}$ These questions remain for future research.

${ }^{20}$ RITTER [1980] surveys anthropologists' attempts to answer this question. In the economics literature, ENGINEER AND BERNHARDT [1992] look at the incentive compatibility conditions between two-period lived generations and ENGINEER, ESTEBAN AND SÁKOVICS [1997] examine the core of a similar OLG model. Generally speaking, it is not incentive compatible for the young to support the old and the core of the model is empty in the absence of institutions. These papers do not examine the more complex relations found in age-group societies. 


\section{References}

ALlais, M. [1947], Economie et Interet, Imprimerie Nationale: Paris.

Auerbach A. AND L. Kotlikoff [1987], Dynamic Fiscal Policy, Cambridge University Press: New York.

Press: Cambridge, MA.

[1998], Macroeconomics: An Integrated Approach, The MIT

AZARIADIS, C. [1993], Intertemporal Macroeconomics, Blackwell: Oxford.

BEAMAN, A. W. [1981], "The Rendille Age-set System in Ethnographic Context:

Adaptation and Integration in a Nomadic Society," unpublished Ph.D. dissertation, Department of Anthropology, Boston University.

Bernardi, B. [1985], Age Class Systems, Cambridge University Press: New York.

BERNHEIM, B.D. AND K. BAGWELL [1988], "Is Everything Neutral?" Journal of Political Economy, 96, 308-338.

, A. SHLEIFER, AND L. Summers [1985], "The Strategic Bequest Motive," Journal of Political Economy, 93, 1045-1076.

Blanchard, O. [1985], "Debt, Deficit, and Finite Horizons," Journal of Political Economy, 93, 223-247.

BROCK, W. [1990], "Overlapping Generations Models with Money and Transactions Costs," pp. 263-295 in: B. Friedman and F. Hahn (eds.), Handbook of Monetary Economics, NorthHolland: Amsterdam.

BULlarD, J. [1992], "Samuelson's Model of Money with n-Period Lifetimes," Economic Quarterly, 78, 67-82.

ENGINEER, M. AND D. BERnhARDT [1992], "Endogenous Transfer Institutions in Overlapping Generations," Journal of Monetary Economics, 29, 445-474.

, J. ESTEBAN, AND J. SÁKOVICS [1997], "Costly Transfer Institutions and the Core in the Overlapping Generations Model", Journal of Economic Behavior and Organization, 32, 287-300.

, E. Roth, AND L. Welling [2004], "Modeling Social Rules and History: The Impact of the Sepaade Tradition on the Rendille of Northern Kenya", mimeo, University of Victoria.

Foner, A. AND D. KERTZER [1978], "Transitions over the life course: lessons from age-set societies", American Journal of Sociology, 83(5), 1081-1104.

GEANAKOPLOS, J. [1987], "Overlapping Generations Models of General Equilibrium," pp. 205-233 in: J. Eatwell, M. Milgate, and P. Newman (eds.), The New Palgrave: General Equilibrium, Norton: New York.

Gokhale, J., L. J. Kotlikoff, J. Sefton and M. Weale [2001], "Simulating the transmission of wealth inequality via bequests," Journal of Public Economics, 79(1), 93-128.

Greenwood, J., N. GunER, AND J. KNOWLES [2003], "More on Marriage, Fertility, and the Distribution of Income," International Economic Review, 44(3).

HAHN, F. AND R. SOlow [1995], A Critical Essay on Modern Macroeconomic Theory, The MIT Press: Cambridge, MA.

Kennedy, P. AND L. Welling [1997], "Production Externalities and the Efficiency of Parental Childcare Choices," Canadian Journal of Economics, 30, 822-834.

KaratZas, I., M. ShUBIK, AND W. SUDDERTH [2002], "A Stochastic Overlapping Generations Economy with Inheritance", Journal of Economics, 77(3), 207-240.

MiLls, J. P. [1926], The Ao Nagas, MacMillan and Co., Limited: London.

OBSTFELD, M. AND K. RogOFF [1996], Foundations of International Macroeconomics, The MIT Press: Cambridge, MA.

RADCLIFFE-BROWN, A. [1929]. “Age Organization - Terminology,” Man, 29, 21. 
Riley, M.W., A. Foner, AND J. WARING [1988], "Sociology of Age," pp. 143-290, in: N. Smelser (ed.), Handbook of Sociology, Sage: Newbury Park, CA.

RitTER, M. [1980], "The conditions favoring age-set organization," Journal of Anthropological Research, 36, 87-104.

Rотн, E. [1993], "A Re-examination of Rendille Population Regulation", American Anthropologist, 95, 597-611.

SAMUELSON, P. [1958], "An Exact Consumption Loan Model of Interest, with or without the Social Contrivance of Money," Journal of Political Economy 66, 467-482.

SPENCER, P. [1997], The Pastoral Continuum, Oxford University Press: New York.

SteWART, F.H [1977], Fundamentals of Age-Group Systems, Academic Press: New York. 
THE AGE-SET MODEL

A collection of groups in a society constitutes an age-set sequence when the groups are governed by rules such that in that society they generate an unbounded number of groups with the following characteristics:

1. The ordering characteristic. There is a total ordering on the groups given by the order in which they begin recruiting.

2. The no-overlapping characteristic. Each group ceases permanently to recruit members before the next one starts.

3. The two-group characteristic. There are always at least two groups in existence.

4. The dissolution characteristic. No group dissolves before one that began recruiting before it.

5. The enrolment characteristic. There is a certain age (the enrolment age) that has the following properties:

No individual joins a group before reaching this age: it is the minimum enrolment age.

Any individual who has not yet joined a group, but who is going to join one, and who has reached this age, joins a group as soon as there is one recruiting members: it is the basic enrolment age.

6. The single membership characteristic. No individual is at any time a member of more than one group.

7. The no-resigning characteristic. A member only leaves a group under one of the following circumstances:

(i) When he leaves the society, or

(ii) When the group is dissolving.

8. The no-rejoining characteristic. A member who has left a group because he has left the society or because the group is dissolving does not again join a group.

STEWART [1977, 28] 
Table 2

\section{THE AGE-GRADE SYSTEM}

An age-grade is one of a finite collection of not less than two rule-sets. The rule-sets are all different from one another, and the collection is totally ordered, say $\mathrm{G}_{1}, \mathrm{G}_{2}, \ldots, \mathrm{G}_{\mathrm{n}}$. The rule-sets are assigned and disassigned to persons by rules that meet the following constraints:

1. The first-grade constraint. No set is assigned to a person before $G_{1}$.

2. The last-grade constraint. No set is assigned to a person after $G_{n}$.

3. The sequential-assignment constraint. If the highest set a person has been assigned is $G_{i}(i=1,2, \ldots, n-1)$, and that person is assigned another set, then the set assigned is $\mathrm{G}_{\mathrm{i}+1}$.

4. The whole-sequence constraint. An individual who has been assigned some $\mathrm{G}_{\mathrm{i}}(\mathrm{i}=1,2, \ldots, \mathrm{n}-1)$ will eventually be assigned $\mathrm{G}_{\mathrm{n}}$ if (but not only if) he survives to the maximum life span.

5. The unique-assignment constraint. No person is at any time assigned more than one set.

6. The no-interval constraint. If a person who is disassigned a set is assigned another, then the two events occur simultaneously.

STEWART [1977, 130] 
Figure 1

Two-period OLG Model

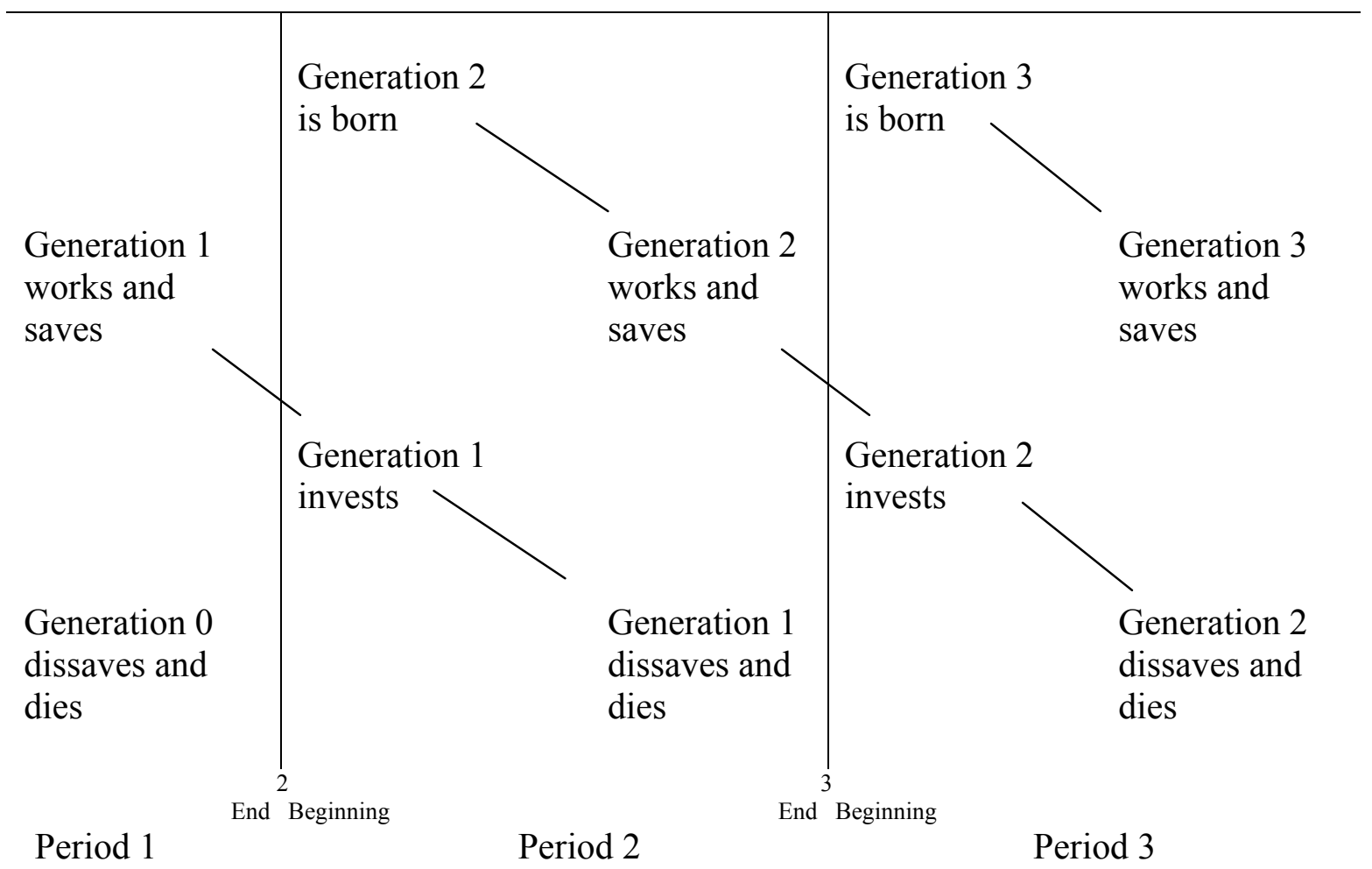

In the life-cycle model, each generation lives for two periods. For example, generation 2 is born at the beginning of time period 2. It earns wages, consumes, and saves at the end of period 2. It invests its assets at the beginning of period 3, consumes, and dissaves at the end of period 3, and then dies. The same life-cycle pattern is experienced by every other generation. Each earns wages and saves when young. Each invests and consumes principal and interest when old.

(modified Figure 2.1 from AUERBACH AND KoTLIKOFF [1998]) 
Figure 2

The Changki Ao Nagas of Northeast India:

A Society with Simple Level Rules

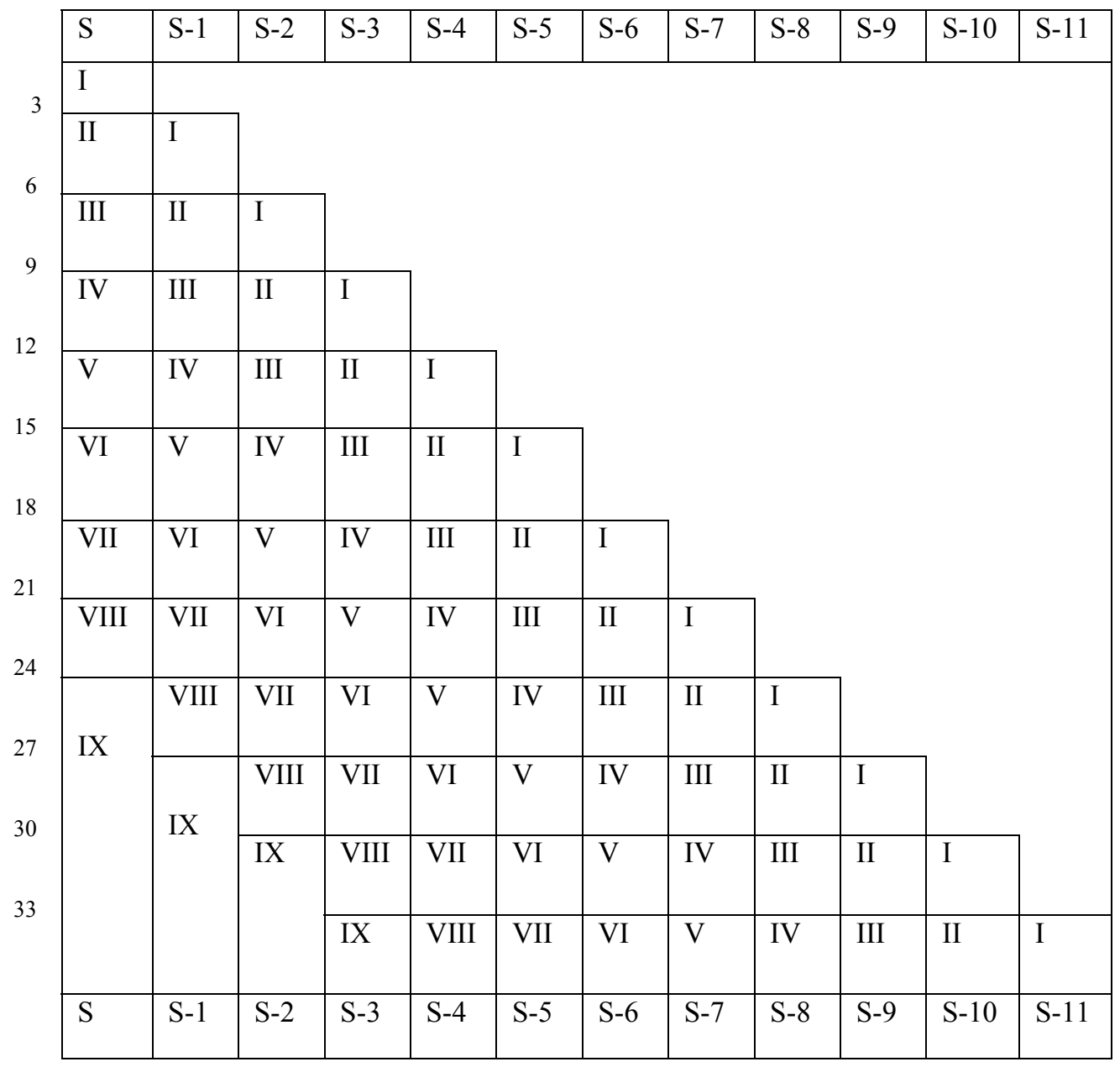

STEWART $[1977,136]$ 


\section{Figure 3}

\section{Formation of Age-Set $S$}

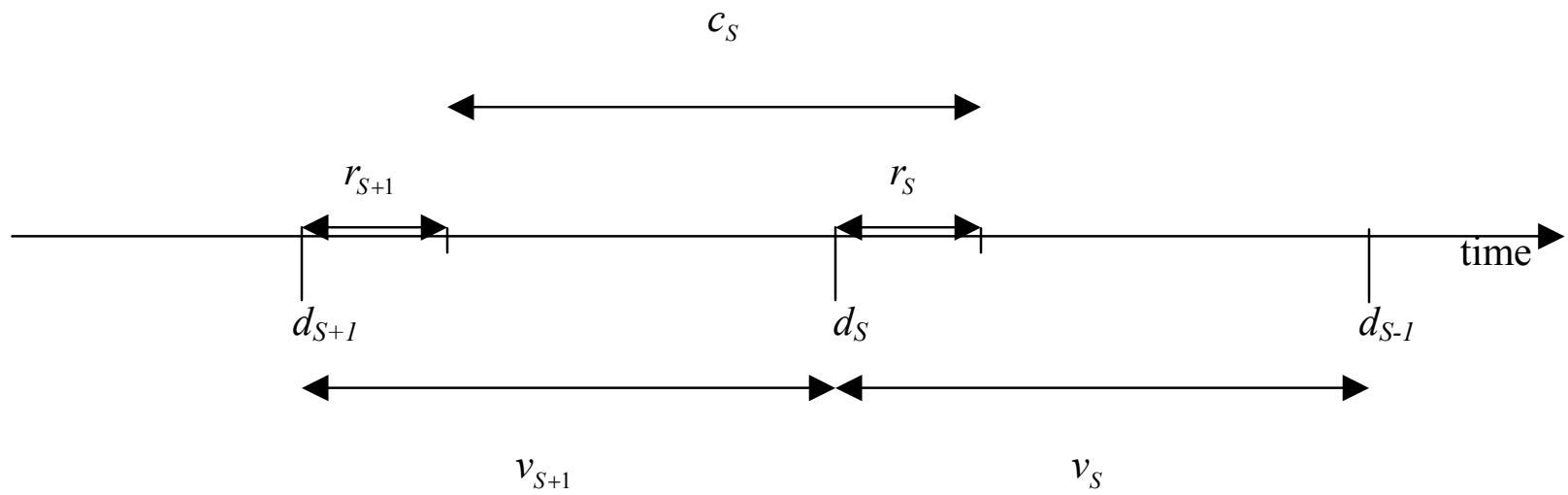

$d_{S}$-- inauguration date for age-set $S$

$v_{S}$-- inauguration interval for age-set $S$

$c_{S}$-- cessation interval for age-set $S$

$r_{S}$-- recruitment period for age-set $S$

(modified figure from STEWART $[1977,35]$ ) 


\section{Merwan H. Engineer \\ Department of Economics \\ University of Victoria \\ Victoria, British Columbia \\ Canada \\ V8W 2 Y2 \\ E-mail: menginee@uvic.ca}

Linda Welling

Department of Economics

University of Victoria

Victoria, British Columbia

Canada

V8W 2 Y2

E-mail: welling@uvvm.uvic.ca 